\title{
Stacked Structure Improves Output Reproducibility of Rubber Force Sensor
}

\author{
Masato Ohmukai', Yasushi Kami1 ${ }^{1}$, Ryo Yawata ${ }^{2}$ \\ ${ }^{1}$ Department of Electrical and Computer Engineering, Akashi College of Technology, Akashi, Japan \\ ${ }^{2}$ Department of Electrical and Electronics Engineering, University of Fukui, Fukui, Japan \\ Email: ohmukai@akashi.ac.jp
}

How to cite this paper: Ohmukai, M., Kami, Y. and Yawata, R. (2016) Stacked Structure Improves Output Reproducibility of Rubber Force Sensor. Journal of Sensor Technology, 6, 75-80.

http://dx.doi.org/10.4236/jst.2016.63006

Received: August 21, 2016

Accepted: September 4, 2016

Published: September 7, 2016

Copyright $\odot 2016$ by authors and Scientific Research Publishing Inc. This work is licensed under the Creative

Commons Attribution International

License (CC BY 4.0).

http://creativecommons.org/licenses/by/4.0/

\begin{abstract}
The electricity-conducting rubber force sensor is an attractive candidate as a low-cost material for tactile sensors. This article shows the evidence that the output reproducibility is largely improved when two identical sheets of the sensors are stacked. The stacked structure may reduce accidental error that is a fatal obstacle in an accurate control system.
\end{abstract}

\section{Keywords}

Rubber Force Sensor, Stacking Structure, Low-Cost Sensor in Control System

\section{Introduction}

Fully automated control systems are widely used in mass production factories since long before. In some cases, a half-automated system is engaged where the operator's decision is required during a process. In other words, an operator should adjust something in such a system. For example, it is difficult to realize fully-automated robot in a nursing care field. So, partly-assisted care robots have been developed steeply nowadays. Tactile sensors can play a role in an interface between an operator and a machine as for touching [1]-[4].

We have been engaged in the development of the electricity-conducting rubber force sensor as a tactile sensor in these years [5], because the conducting rubber sheet is so cheap and easy to handle. We investigated the some kinds of electrodes on the rubber force sensor and found that aluminum deposited in vacuum was suitable because the dynamic range is the largest: until $150 \mathrm{~N}(44 \mathrm{~mm} \times 44 \mathrm{~mm}$ square area).

We further found that radiation heating during the vacuum deposition deteriorated the rubber characteristics [6]. The standard deviation of the output voltage becomes 
large with this deposition method. We have then adopted two sheets of aluminum foil attached to the both side, to reduce the standard deviation in the convergent output voltage. The dispersion of the convergent value was not small enough to be applied to the commercial use.

The rubber force sensor can sense the compression force between the both sides in the form of the reduction of electrical resistance across the both sides. This kind of sensor has a great advantage of low cost but the annoying disadvantage of poor reproducibility in the resistance at the same time. The dispersion of output signal at the same applied force to the sensor has to be an obstacle to use the output signal for a feedback control.

We propose the stacked structure of the sensors in this article and expect the reduction of the output dispersion, resulting in more accurate estimation of the force. Since the large output dispersion is an inherent fault of the rubber sensor, the improvement of this disadvantage will give a large impact on pressure-sensor technologies.

\section{Experimental Details}

The electric-conducting rubber has the structure that conducting particles are dispersed in a rubber matrix. Two kinds of rubber sheet sensors are known depending on a particle substance: metal and carbon. Generally speaking, the electrical resistance reduces when the compression force is applied to the rubber sheet, because the path for electrical current forms following the particles are contact each other by the applied force. The change in the electrical resistance is detected as a voltage change. The response is quite different in these two types. The former shows a step-like response as if there is a certain threshold, while the latter shows a gradual change in resistance by the applied force. In our research, the goal is the estimation of the applied force on the basis of the output voltage; hence the latter type is suitable to apply to a force sensor.

Our previous research shows that the standard deviation of the output voltage is smallest when the electrode of the sensor is aluminum foil just attached to the rubber sheet. Therefore, aluminum foil was adopted in this experiment. The square aluminum foil having a little bit smaller area than the rubber sheet was put on a surface, and only one end of the square was fixed with adhesive tape. On the back side of the rubber sheet, another aluminum foil was put and fixed in the same way on the other end. The stacked structure was formed by the superposition of the rubber sheet with aluminum foil electrode on both sides alternatively. The area of the rubber sheets was a square of 9 $\mathrm{cm}^{2}$.

The sensor was located on a table and a weight was put on the sensor. An iron plate was inserted between the weight and the rubber sensor, in order to avoid non-uniform force to be loaded on the sensor. Since the weight was 100, 300 or 500 grams, the weight of the iron plate was light enough to be ignored. The three kinds of weight give the forces of $0.98,2.9$, and $4.9 \mathrm{~N}$, respectively.

The electric circuit for the detection is shown in Figure 1. The element of $r$ in the figure corresponds to the rubber sheet sensor, the electric resistance of which varies 


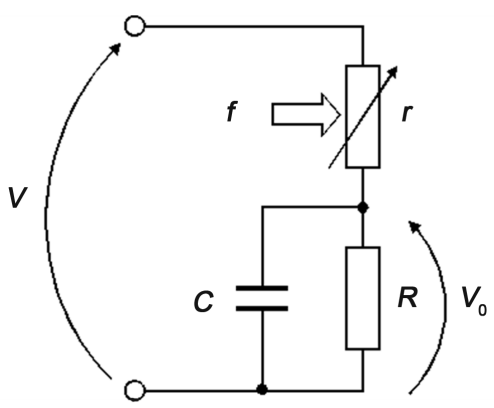

Figure 1. Detecting circuit of the output. The rubber sensor was indicated $r$ in the figure.

depending on the force; it reduces as the applied force increases. Direct current voltage of $5 \mathrm{~V}$ was applied across the left terminals indicated $\mathrm{V}$ in the figure. The load resister $\mathrm{R}$ $(90 \mathrm{k} \Omega$ ) is connected to the rubber sensor in series. The capacitor of $4.9 \mu \mathrm{F}$ was aimed to shunt high-frequency noise.

\section{Results}

We measured the time response on output voltage as for a single rubber sheet sensor. The results are shown in Figure 2; the loaded weights were 100, 300, and $500 \mathrm{~g}$, respectively. The origin of the time was set to the point where the weight was put on the sensor. They show that the transient region is stretched under $0.3 \mathrm{~s}$. Each figure shows 50 trials of the experiments. The convergent outputs show poor reproducibility in all cases.

We next show the similar results in Figure 3 when the two rubber sheet sensors were stacked. The transient region is similar to the previous results. The convergent output voltage is higher because the two outputs voltages are simply added. The noticeable point is that the dispersion of the convergent outputs becomes smaller in all three cases.

The dispersion of the convergent outputs is surely annoying for the estimation of the applied force from the output voltage. It is ideal that there is no dispersion in the convergent output, but it would be actually unhopeful to eliminate the dispersion using such a low-cost device. These results clearly show the effectiveness of the stacked structure to the reduction of dispersion in the convergent output.

\section{Discussion}

Each experiment included 50 trials, where the convergent output is dispersed as described in the previous section. The averaged output through 50 cases is shown in Figure 4 as a function of applied weight. The average out-put increases monotonically with the applied weight in both cases. It is worth noting that the stacked structure gives more proportional relationship between the average output and the applied weight. It can be noticed that the average output from the stacked structure is about twice as large as that from the single structure, for the weight of $500 \mathrm{~g}$. The absolute value of the output voltage, however, depends strongly on the sample, so it does not mean that the stacking enhances the sensitivity. Then the absolute value should be calibrated for a practical use. 



Figure 2. The transient output voltage of 50 trials for a single sensor. The applied load was $100 \mathrm{~g}$ (top-left), $300 \mathrm{~g}$ (bottom-left) and $500 \mathrm{~g}$ (bottom-right), respectively.
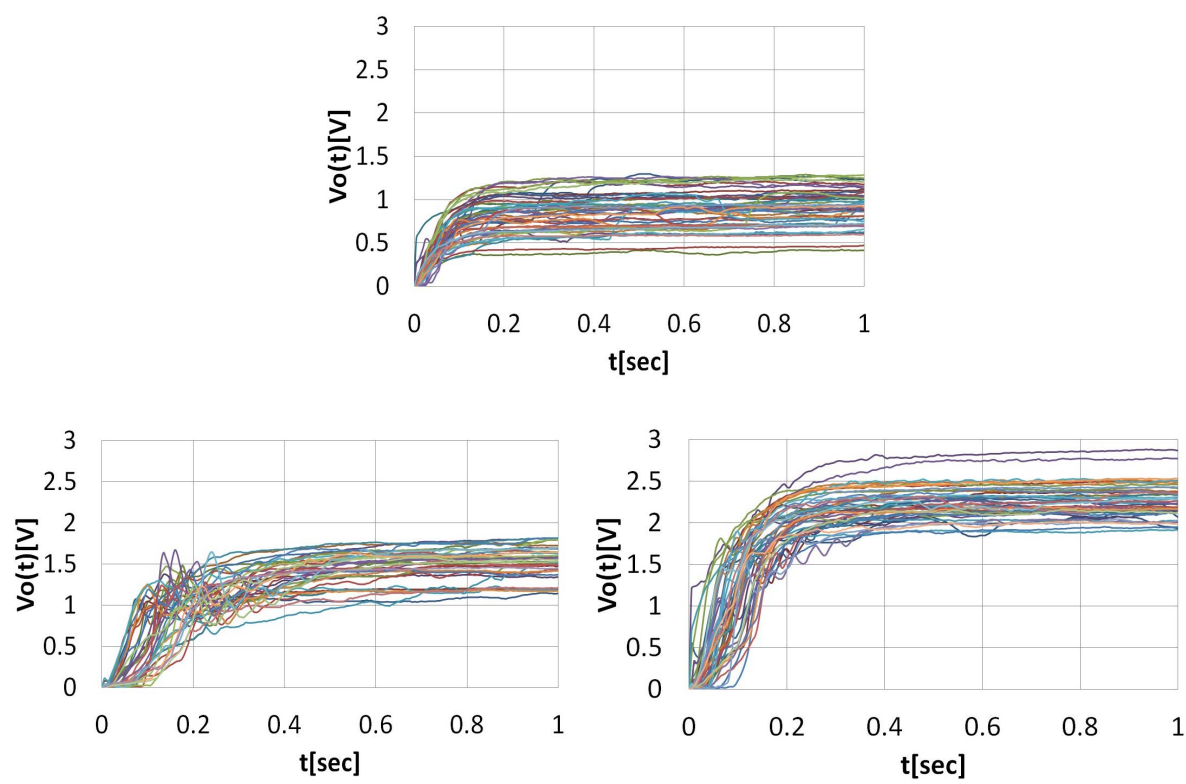

Figure 3. The transient output voltage of 50 trials for a staced sensor. The applied load was $100 \mathrm{~g}$ (top-left), $300 \mathrm{~g}$ (bottom-left) and $500 \mathrm{~g}$ (bottom-right), respectively.
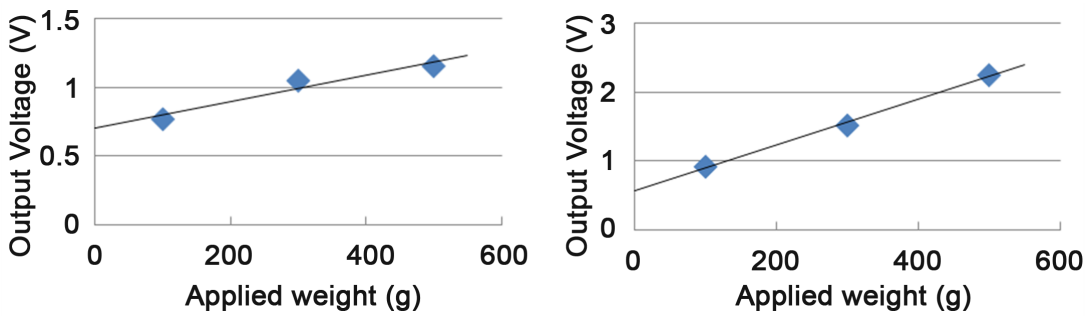

Figure 4. The average output voltage of the convergent value: a single sensor (left) and a stacked sensor (right). 
We next discuss the dispersion in convergent output voltage. The absolute errors for the maximum and minimum output separated from the average value are shown in Figure 5. It can be found that the stacking reduced the errors in both figures. Basically the error declines with the applied weight in general. From these evidences, the stacking drastically suppresses the extreme error.

We treat the error from the aspect of the standard deviation. The obtained standard deviation is shown in Figure 6. In this figure, the standard deviation was taken as the relative value to the average convergent output voltage. The figure well evidences that the relative standard deviation is reduced to a large extent by the stacking.

We now speculate the reason for the improvement of the characteristics by the stacking. The large dispersion of the convergent output voltage inherently derived from the molecular structure and the state of the embedded carbon particles in the rubber matrix. It can be well accepted that this inherently causes the large dispersion in the convergent output voltage. By stacking the rubber sheet sensor, the accidental errors can be compensated each other and then the error possibly reduces by the stacking as a result. It is assumed that more stacking can reduce the accidental errors.

The absolute value of the electric resistance strongly depends on this microscopic structure in nature. It is quite difficult to control the conformation of molecular ordering in polymer materials. We showed that the stacking reduces the dispersion of convergence output evidently. This reduction surely contributes to the more precise control. In this way, one can make the most of the biggest merit of the low-cost pressure sensor.
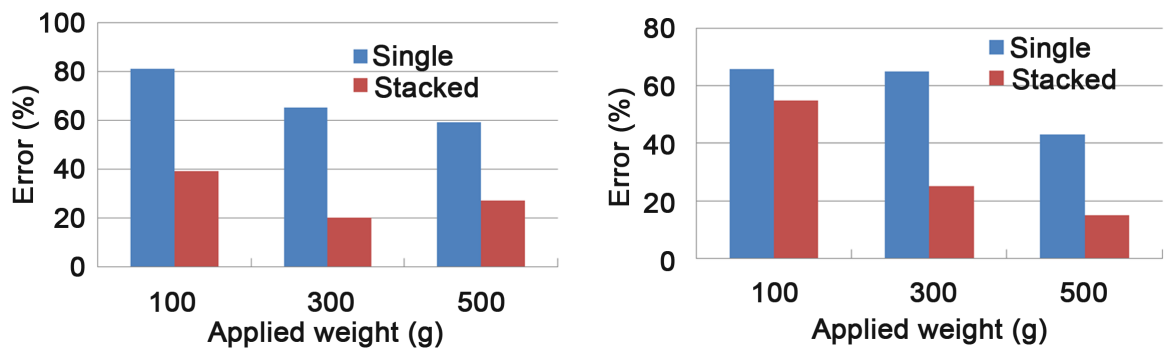

Figure 5. The error of the maximum (left) and minimum (right) convergent value apart from the average output voltage, respectively.



Figure 6. The standard deviation relative to the convergent average output voltage. 


\section{Conclusion}

The electric-conducting rubber-sheet pressure sensor has a great advantage of low cost but the big problem of the large dispersion of convergent output at the same time. In order to suppress the dispersion, the stacking structure was introduced. Our experimental results show that the dispersion was suppressed successfully. In addition, it was found that the relation between the weight loaded on the sensor and the output voltage becomes more proportional by the stacking. It is expected that the more number of stacking might reduce the dispersion further for practical applications.

\section{Acknowledgements}

This work was partially supported by a Grant-in-Aid for Scientific Research No. 16K01430 from the Ministry of Education Science, Sports, and Culture, for which the authors are grateful.

\section{References}

[1] Lee, M.H. and Nicholls, H.R. (1999) Review Article Tactile Sensing for Mechatronics-A State of the Art Survey. Mechatronics, 9, 1-31. http://dx.doi.org/10.1016/S0957-4158(98)00045-2

[2] Li, Z., Hsu, P. and Sastry, S. (1989) Grasping and Coordinated Manipulation by a Multifingered Robot Hand. International Journal of Robotics Research, 8, 33-50. http://dx.doi.org/10.1177/027836498900800402

[3] Berger, A.D. and Khosla, P.K. (1991) Using Tactile Data for Real-Time Feedback. International Journal of Robotics Research, 10, 88-102. http://dx.doi.org/10.1177/027836499101000202

[4] Schmidt, P.A., Mael, E. and Wurtz, R.P. (2006) A Sensor for Dynamic Tactile Information with Applications in Human-Robot Interaction \& Object Exploration. Robotics and Autonomous Systems, 54, 1005-1014. http://dx.doi.org/10.1016/j.robot.2006.05.013

[5] Ohmukai, M., Kami, Y. and Matsuura, R. (2012) Electrode for Force Sensor of Conductive Rubber. Journal of Sensor Technology, 2, 127-131. http://dx.doi.org/10.4236/jst.2012.23018

[6] Ohmukai, M., Kami, Y. and Ashida, K. (2013) Conducting Rubber Force Sensor: Transient Characteristics and Radiation Heating Effect. Journal of Sensor Technology, 3, 36-41. http://dx.doi.org/10.4236/jst.2013.33007 
Submit or recommend next manuscript to SCIRP and we will provide best service for you:

Accepting pre-submission inquiries through Email, Facebook, LinkedIn, Twitter, etc. A wide selection of journals (inclusive of 9 subjects, more than 200 journals)

Providing 24-hour high-quality service

User-friendly online submission system

Fair and swift peer-review system

Efficient typesetting and proofreading procedure

Display of the result of downloads and visits, as well as the number of cited articles

Maximum dissemination of your research work

Submit your manuscript at: http://papersubmission.scirp.org/ 